\title{
An Incremental Approach to Scheduling during Overloads in Real-Time Systems*
}

\author{
Pedro Mejía-Alvarez ${ }^{\dagger}$ \\ CINVESTAV-IPN. Sección de Computación \\ Av. I.P.N. 2508, Zacatenco. \\ México, DF. 07300 \\ pmejia@computacion.cs.cinvestav.mx
}

\author{
Rami Melhem, Daniel Mossé \\ Computer Science Department \\ University of Pittsburgh \\ Pittsburgh, PA 15260 \\ melhem,mosse@cs.pitt.edu
}

\begin{abstract}
In this paper we propose a novel scheduling framework for a real-time environment that experiences dynamic changes. This framework is capable of adjusting the system workload in incremental steps under overloaded conditions such that the most critical tasks in the system are always scheduled and the total value of the system is maximized. Each task has an assigned criticality value and consists of two parts, a mandatory part and an optional part. A timely answer is available after the mandatory part completes execution and its value may be improved by executing the entire optional part. Optional parts can be discarded in overloaded conditions.

The process of selecting optional parts to discard while maximizing the value of the system requires the exploration of a potentially large number of combinations. Since this process is too time consuming to be computed on-line, an approximate algorithm is executed incrementally whenever the processor would otherwise be idle, progressively refining the quality of the solution. This criteria allows the scheduler to handle overloads with low cost while maximizing the use of the available resources and without jeopardizing the temporal constraints of the most critical tasks in the system. Simulation results show that few stages of the algorithm need to be executed for achieving a performance with near-optimal results.
\end{abstract}

\section{Introduction}

The use of complex and dynamic real-time systems is nowadays becoming common for the management and control of a variety of applications such as manufacturing, industrial automation systems, space or avionics and telecommunications systems. In a critical real-time system, each task must complete and produce correct results by the specified deadline. Failure to conform with any timing constraint is considered

\footnotetext{
* This work has been supported by the Defense Advanced Research Projects Agency through the FORTS project (Contract DABT63-96-C-0044)

tWork done while this author was visiting the Information Sciences and Telecommunications Department, University of Pittsburgh
}

a catastrophic failure. In order to guarantee that the timing constraints will be satisfied, it is necessary that the resource requirements for all tasks in the system be known and that resources be available in a timely manner. Therefore, the resources must be reserved for worst-case execution time of tasks to provide absolute guarantees.

Traditionally, the resource scheduling problem for real-time tasks is to generate a feasible schedule or to verify if a given scheduling policy can meet the timing requirements of a specific set of tasks. In practice, however, real-time environments experience frequent changes in workloads, caused by new task arrivals or tasks that leave the system after finishing their execution. The problem with accepting new tasks in the system is that they may result in an overload and cause some of the tasks already in the system to miss their deadlines. Under such overload conditions, we may augment the available resources or reject some tasks (or both).

In this paper we study the problem of scheduling dynamic tasks in an overloaded single processor environment, where new tasks arrive or leave the system at arbitrary instances of time. A framework is proposed for adjusting the system workload incrementally by relating the criticality value $[2,3]$ of the tasks to the resource allocation problem. The identification of feasible options that maximize an optimality criteria (expressed as the total value of the system) requires the exploration of a potentially large combinatorial space of solutions. Our approach to solve this problem is based on an on-line Incremental Server (INCA), which searches feasible solutions by executing a sequence of approximate algorithms. At each approximate algorithm execution, the load is adjusted and the quality of the solution is refined. The minimum number of approximate algorithms executed produces a feasible but suboptimal solution that can be incrementally improved if more approximate algorithms can be executed. Functions with this property are called incrementally precise functions or incremental processes.

We consider real-time tasks that consist of mandatory parts and optional parts for refining the result of the mandatory parts. Systems exhibiting this behavior include (1) multimedia systems that receive, enhance or transmit audio, video or images 
and process this information for specific intervals of time, (2) process control systems with sensors and actuators that are activated by changing environmental conditions and (3) real-time database query processing systems. For systems such as these, our approach is to produce approximate solutions that can be progressively refined, when the exact solutions cannot be produced in time, due to overloaded conditions.

The remainder of this paper is organized as follows. In Section 2 related models and previous work are reviewed. In Section 3, the task model used in this paper is defined. In Section 4 , the overload scheduling problem is formulated. In Section 5 , the INCA server is described and in Section 6 we analyze the merit of the incremental execution of the INCA server, and compare its performance against a non incremental server. In Section 7, simulation results are presented to show the performance of the INCA Server and to give insight into its effectiveness in handling overload conditions. Finally, Section 8 presents concluding remarks.

\section{Related Work}

In a dynamic real-time environment, even when the system is properly designed and sized, a transient overload can occur for different reasons, such as changes in the environment, simultaneous arrivals of asynchronous events, faults of peripheral devices, or system exceptions[5]. The worst consequence that may happen is that some critical tasks in the system miss their deadlines, jeopardizing the correct/safe behavior of the system. As all systems have finite resources, their ability to execute a set of periodic and aperiodic tasks while meeting the temporal requirements is limited. Clearly, overload conditions arise if the system has to process more new tasks than the available set of resources can handle.

In the real-time literature several scheduling algorithms have been proposed to deal with overloaded systems. The development of the Best-Effort algorithm [14] introduced a rejection policy for overloaded systems based on removing tasks with the minimum value density. The Best-Effort approach basically behaves as the Earliest Deadline First [12] when the system is underloaded and chooses the subset of tasks that maximize the value of the computation per unit of time (value density) when the system is overloaded. The Alpha effort [9] introduced the concept of time-valued functions, which associate a value according to the task finishing time. The function presents a drop in value after the deadline has passed, and beyond a certain time the value drops to zero.

The problem of selecting tasks for rejection in an overloaded system is also considered in [8], where random criticality values are assigned to tasks. An approximate algorithm incorporates simulated annealing to deal with the problem of selecting a feasible solution within the large combinatorial space of permutations. The RED (Robust Earliest Deadline) algorithm [4] deals with aperiodic tasks in overloaded environments, combining criticality-based scheduling, deadline tolerance (the amount of time by which a task is permitted to be late) and resource reclaiming. It is able to predict not only deadline misses but also the size of the overload, its duration, and its impact on the system. However, the strategy for handling the overload is to reject the least-valued task. Other approaches for handling overload focuses on providing a less stringent guarantees for temporal constraints. In [10] some instances of a task are allowed to be skipped entirely. The skip factor determines how often instances of a given task may be left unexecuted. A best effort approach is introduced in [7], aiming at meeting $k$ deadlines out of $n$ instances of a given task. However, it is assumed that the value of the tasks in the system is proportional to their computation time, provided that they complete by their deadlines.

Many of these techniques (e.g., [10,7]) assume that a task's output is of no value if it is not executed completely. In contrast, in the Imprecise Computation(IC) model the task's output has some value even if a partial or approximate result is produced $[1,13]$. In the IC model, every real-time task is composed of a mandatory part and an optional part. A timely answer is available after the mandatory part completes execution; moreover, the longer the optional part executes, the higher the value of the task (i.e., the higher the quality of the result). However, the IC model uses an error function as a metric to evaluate the performance of the system. In [13] an error function is defined to be inversely proportional to the total amount of time that the optional parts execute. An optimal schedule corresponds to the one where the total error of the system is minimized. In the IC model the shape of the error functions and policies for scheduling optional parts are crucial in maximizing the performance of the system.

From previous work we have learned that: (a) many scheduling algorithms have been developed for overloaded conditions, but few research work studied in practice how far from optimal is the performance of their algorithms and their complexity (reference [11] provided a measure for the performance of their D-over algorithm using a metric called competitive factor; this metric denotes the cumulative value of the system compared with the optimal value obtained by a clairvoyant scheduler that knows the entire task set a priori);(b) In most developed algorithms, the criteria for rejection in overloaded conditions is to select the lesser-valued tasks, a strategy that clearly yields low cost solutions but may lead to a situation with underutilized resources and a resulting system with poor performance; and (c) the time-value function of [9] or the error functions of the IC model [13] are difficult to obtain, and performance may be degraded if the system designers are not familiar with the functions that represent the applications at hand. Although our model is similar to the IC model, we do not use error functions but performance metrics that are largely available, such as utilization and criticality $[2,3]$.

\section{Task Model}

In our framework we consider periodic preemptive tasks running on one processor. Tasks are independent and have no 
precedence constraints. Each task $\tau_{i}$ arrives in the system at time $a_{i}$. The life-time of each task $\tau_{i}$ consists of a fixed number of instances $r_{i}$. After the execution of $r_{i}$ instances, the task leaves the system ${ }^{1}$. The time interval between the arrival of the first instances of two consecutive tasks $\tau_{x}$ and $\tau_{y}$ is defined as $l_{x y}=a_{y}-a_{x}$. We assume that the tasks characteristics (e.g., computation time, period, deadline and criticality) are known at arrival time. Each task $\tau_{i}$ is decomposed into a mandatory part $M_{i}$ followed by an optional part $P_{i}$. The use of the imprecise computation model is not restrictive in the sense that each task may have only an optional part and no mandatory part. In this model, $T_{i}$ is the period and $C_{i}$ is the worst case computation time of task $\tau_{i}$. Each execution time $C_{i}$ consists of a mandatory part of length $m_{i}$ and an optional part of length $p_{i}$ (i.e., $C_{i}=m_{i}+p_{i}$ ). The mandatory part $M_{i}$ must execute to completion in order to produce an acceptable and usable result. The optional part $P_{i}$ can execute only after the completion of the mandatory part $M_{i}$. However, a partially executed optional part or an optional part that misses its deadline is of no value to the system ( $0 / 1$ constraint). The task $\tau_{i}$ meets its deadline if its mandatory part completes by its deadline. It is assumed that the set of mandatory parts can never cause an overload in the system. Each task has an associated criticality value $v_{i}$, which denotes its importance within the system ${ }^{2}$. The Earliest Deadline First[12] priority assignment scheme will be considered.

\section{Formulation of the Problem}

In overloaded conditions, the scheduler should be able to guarantee the timing constraints of all mandatory parts at every periodic task invocation and to select optional parts for exclusion from the schedule while maximizing the performance of the system. If the criticality of each task is proportional to its computation time, the decision of excluding optional parts must be based only on maximizing the usage of the resources (e.g., CPU time). In the more general case, where criticality and computation time are not directly related, we would like to exclude the less critical optional parts and maximize the total value of the system. Therefore, the problem can be formulated as follows. If a new task $\tau_{i}$ that arrives in the system at time $a_{i}$ causes an overload, the problem is (a) to determine whether or not $\tau_{i}$ can be accepted in the system without interfering with the deadlines of the mandatory parts of any task already in the system, (b) if accepted, at what time $\tau_{i}$ should be dispatched?, (c) what optional parts (if any) should be excluded such that an optimality criteria is satisfied and (d) while searching for a solution, how can we maximize the usage of the resources and the performance of the system with a reasonable low cost?

Each task in the system accrues an accumulated value upon executing a number of optional parts during its life-time. Our objective is to maximize the accumulated value obtained after scheduling the set of optional parts for the complete duration of

\footnotetext{
${ }^{1}$ We assume that some tasks that leave the system may return at a later time.

${ }^{2}$ Methods to derive this criticality values are proposed in $[2,3,14]$.
}

the schedule. The accumulated value will be evaluated in terms of utilization or criticality as follows. Let us define $C U(I)$ and $C V(I)$ as the cumulative utilization and cumulative criticality potentially achieved by the set of optional parts that execute during the interval of time $I$.

The cumulative utilization achieved is computed by,

$$
C U(I)=\sum_{i=1}^{n} I * \frac{p_{i}}{T_{i}}
$$

The cumulative criticality achieved is computed by,

$$
C V(I)=\sum_{i=1}^{n} I * \frac{v_{i}}{T_{i}}
$$

For the interval of time between two consecutive arrivals $a_{x}$ and $a_{y}$ the accumulated value can be formulated as $C U\left(l_{x y}\right)$ and $C V\left(l_{x y}\right)$ for utilization and criticality respectively. At $a_{x}$, the goal is to select the optional parts that maximize $C U\left(l_{x y}\right)$ or $C V\left(l_{x y}\right)$. This selection requires the searching of a usually large search space.

\subsection{Definition of the Search Space}

Consider $n$ tasks, $\tau_{1}, \ldots, \tau_{n}$ such that $\sum_{i=1}^{n} \frac{m_{i}}{T_{i}} \leq 1$ and $\sum_{i=1}^{n} \frac{m_{i}+p_{i}}{T_{i}}>1$. That is, all mandatory parts can be accepted for execution but not all optional parts can be accepted for execution.

Let $S$ be the search space containing all combinations (both feasible and non-feasible) of optional tasks. More specifically, a search space is defined as $S=\cup_{k=0}^{n} S_{k}$ where $S_{k}=$ $\left\{\left(x_{1}, \ldots, x_{n}\right) ; \sum_{i=1}^{n} x_{i}=k\right\}$ where $x_{i}=0$ means that the optional part of $\tau_{i}$ is discarded and $x_{i}=1$ means that the optional part of $\tau_{i}$ is chosen for execution. Note that for any $k \in\{0, . ., n\}, S_{k}$ is a set of elements containing all (feasible and non-feasible) combinations resulting from including $k$ optional parts for execution and that $\left|S_{k}\right|=\frac{n !}{k !(n-k) !}$. Any

\begin{tabular}{|c|c|}
\hline Set & Search Space \\
\hline $\begin{array}{l}S_{4} \\
S_{3} \\
S_{2} \\
S_{1} \\
S_{0}\end{array}$ & $\begin{array}{l}\{1,1,1,1\} \\
\{1,1,1,0\}\{1,1,0,1\}\{1,0,1,1\}\{0,1,1,1\} \\
\{1,1,0,0\}\{1,0,1,0\}\{1,0,0,1\}\{0,1,1,0\}\{0,1,0,1\}\{0,0,1,1\} \\
\{1,0,0,0\}\{0,1,0,0\}\{0,0,1,0\}\{0,0,0,1\}\end{array}$ \\
\hline
\end{tabular}
element in $S_{k}$ includes for execution exactly $k$ optional parts.

Table 1. Search space for four tasks.

The structure of the search space $S$ is shown in Table 1 through an example with 4 tasks. For example, $\{1,1,0,0\}$ is the element in which $p_{1}$ and $p_{2}$ are included for execution and $p_{3}$ and $p_{4}$ are discarded.

\subsection{Definition of the Objective Functions}

Each element in the search space will be evaluated in terms of utilization or criticality, using the objective functions $\mu(s)$ and $\gamma(s)$ respectively, where $s=\left\{x_{1}, \ldots, x_{n}\right\} \in S$. The objective functions are defined as follows. 
- $\mu(s)$ : In this function we add the utilization of the set of optional parts in an element $s \in S$ to the total utilization of all mandatory parts.

$$
\mu(s)=\sum_{i=1}^{n} \frac{m_{i}}{T_{i}}+\sum_{j=1}^{n} x_{j} \frac{p_{j}}{T_{j}}
$$

$\mu(s)$ denotes the utilization of the system, after choosing some optional parts for execution. For example, for $s=$ $\{0,1,1,0\}, \mu(s)=\sum_{i=1}^{4}\left(\frac{m_{i}}{T_{i}}\right)+\frac{p_{2}}{T_{2}}+\frac{p_{3}}{T_{3}}$.

- $\gamma(s)$ : In this function we compute the criticality per period achieved after including for execution a set of optional parts in an element $s \in S$, recalling that $v_{i}$ is the criticality of task $\tau_{i}$

$$
\gamma(s)=\sum_{i=1}^{n} x_{i}\left(\frac{v_{i}}{T_{i}}\right)
$$

For example, for $s=\{0,1,1,0\}, \gamma(s)=\frac{v_{2}}{T_{2}}+\frac{v_{3}}{T_{3}}$

Note that if the tasks $\left\{\tau_{1}, \ldots, \tau_{n}\right\}$ are to execute during an interval $I$, the choice of $s$ that maximizes $\mu(s)$ and $\gamma(s)$, also maximizes $C U(\mathrm{I})$ and $\mathrm{CV}(\mathrm{I})$ respectively.

\subsection{Feasibility Test}

To evaluate the feasibility of each element in the search space we apply an utilization-based test (UBT). The utilization-based test has been chosen because of its simplicity and because it can be used for scheduling policies such as EDF.

For each element $s=\left\{x_{1}, \ldots, x_{n}\right\}$ of the search space $S$ the utilization-based test is defined by,

$$
U B T(s)= \begin{cases}\text { true } & \text { if } \mu(s) \leq 1 \\ \text { false } & \text { otherwise }\end{cases}
$$

Note that, when choosing a feasible solution, the utilization of the optional parts $\left(U_{p}=\sum_{i=1}^{n} x_{i} \frac{p_{i}}{T_{i}}\right)$ must satisfy: $U_{p} \leq$ $1.0-U_{m}$, where $U_{m}=\sum_{i=1}^{n} \frac{m_{i}}{T_{i}}$. Also, any single optional part with utilization $\frac{p_{i}}{T_{i}}$ greater than $1.0-U_{m}$ can be discarded without any further test.

\subsection{The Optimization Problems}

Our first optimization problem is related to shedding a number of optional parts that maximizes the utilization of the system. This objective favors a solution in which the utilization of the workload is maximized without considering the number of optional parts to be shed. Our second optimization problem assumes that different criticality values are associated with optional parts, therefore we are interested in maximizing the total value obtained after a number of optional parts are shed.

The optimization problems are formally described as follows,
- Maximize the utilization. The aim of this objective is to find a feasible element $s \in S$ such that the utilization in the system is maximized. That is

$$
\begin{array}{cc}
\text { maximize } & \mu(s) \\
\text { subject to } & \mathrm{UBT}(\mathrm{s})
\end{array}
$$

Let $U^{\max }$ be the value of $\mu(s)$ obtained by solving this optimization problem.

- Maximize the value. Maximizing the value requires to find a feasible element $s \in S$ such that $\gamma(s)$ is maximized. That is

$$
\begin{array}{cc}
\text { maximize } & \gamma(s) \\
\text { subject to } & \text { UBT(s) }
\end{array}
$$

Let $V^{\max }$ be the value of $\gamma(s)$ obtained by solving this optimization problem.

The optimization problems consist of maximizing the value of the system at the instant of time at which a new arrival causes an overload in the system. By achieving the optimality criteria, whenever a new task arrives or departs from the system, we intend to maximize the accumulated value (CU(I) or $\mathrm{CV}(\mathrm{I})$ ) obtained after scheduling the entire set of tasks for the complete duration of the schedule.

\section{The Incremental Scheduling Server: INCA}

The incremental scheduling server is an extension of the earliest deadline first scheduling algorithm (EDF[12]). In response to transient overload requests, the INCA Server adjusts the load of the system by executing a sequence of approximate algorithms, $A P(0), \ldots, A P(n)$ to determine which optional parts to shed in order to satisfy our optimality criteria. The algorithms are such that $A P(i)$ may obtain a solution closer to optimal than $A P(i-1)$ but with longer execution time. The INCA Server is activated whenever the feasibility test (UBT) detects an overload caused by the arrival of a new task in the system. The INCA Server first executes the approximate algorithm $\mathrm{AP}(0)$ to eliminate the overload. The solution provided by $\mathrm{AP}(0)$ allows the scheduler to disable temporarily the execution of some optional parts ${ }^{3}$, while providing a low cost non-optimal solution. The slack-time ${ }^{4}$ introduced in the system by the removal of the overload is used by the scheduler to execute approximate algorithms $\operatorname{AP}(\mathbf{k})($ for $k=1, \ldots, n)$, progressively refining the quality of the solution.

If during the execution of the INCA Server a new task arrives or a task leaves the system, it will re-start its execution, taking into account the modified load of the system.

\footnotetext{
${ }^{3}$ Note that after the execution of each approximate algorithm some optional parts may be disabled temporarily, but not discarded. The reason for this is that at each approximate algorithm we may find different solutions involving different optional parts to execute.

${ }^{4}$ Slack-time is defined as the time at which the processor is not executing any task.
} 


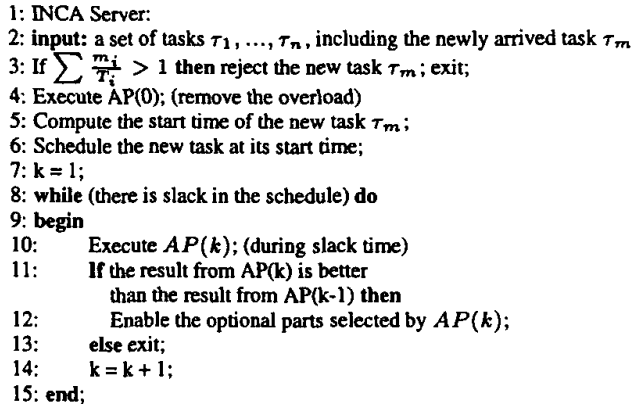

Figure 1. Incremental Server (INCA)

The INCA Server stops its execution when (a) there is no more slack in the schedule to execute additional $\mathrm{AP}(\mathrm{k})$ algorithms, or (b) the result of $\mathrm{AP}(\mathrm{k})$ is not better than the result of $\mathrm{AP}(\mathrm{k}-1)$. The INCA Server is described in Figure 1.

\subsection{Methodology for Handling Overload}

In this section, a methodology for handling overload conditions is introduced.

1. Activating the Incremental Server. The INCA server is activated if a new task, $\tau_{m}$, arrives in the system and causes an overload. Our feasibility test (UBT) detects this condition. After detecting the overload, AP(0) is executed ${ }^{5}$ and some optional parts are chosen to be discarded for removing the overload. The INCA server is also activated when a task leaves the system. In this case, the approximate algorithms AP(k) (for $k=0, \ldots, n)$ are executed incrementally to satisfy the optimality criteria for the new set of optional parts in the system.

2. Scheduling the new task. After removing the overload from the system, the newly arrived task can be scheduled. However, regardless of the priority of the newly arrived task, if the task is accepted it may not be scheduled at its arrival time because it may cause some missing deadlines, even after executing AP( 0$)$. This is because, at the instant of the new arrival, we may choose optional parts that (a) already finished their current execution, or (b) have been preempted while executing their optional parts. The resulting utilization cannot be immediately subtracted from the total processor load because the discarded optional parts could already have delayed the execution of other tasks. As a consequence, to keep the feasibility test consistent, the utilization of a discarded optional part can be subtracted from the total load only at the end of its current period. Thus, the new task should wait until the end of the longest period of all preempted tasks ${ }^{6}$. Clearly, the tasks that

\footnotetext{
${ }^{5}$ We assume that the execution times of AP(0) and UBT are negligible.

${ }^{6}$ Computing analytically the best time at which it is possible to accept the new task may involve some additional run-time overhead[6]. Therefore we
}

have not already started the execution of their optional parts allow the utilization to be subtracted immediately.

3. Execution of $\mathbf{A P}(1), \ldots, \mathbf{A P}(\mathbf{n})$. After removing the overload through $A P(0)$, an increase in the slack available in the system is expected. $A P(1)$ is then executed on the slack existing in the system after the execution of $\mathrm{AP}(0)$. Analogously, $A P(k)$ (for $k=2, \ldots, n$ ) is executed on the slack existing in the system after the execution of the previous $A P(k-1)$. The INCA Server executes the approximate algorithms AP(1), ... $\mathrm{AP}(\mathrm{n})$ incrementally. After executing each $A P(k)$, the workload of the system is adjusted by enabling and disabling some optional parts. Since the INCA Server executes on the slack available in the system, it will execute as many approximate algorithms as possible.

Algorithm AP(k) may yield better solutions than $\mathrm{AP}(\mathrm{k}-1)$ but at the cost of higher execution times. However, the execution of each $\mathrm{AP}(\mathrm{k})$ may increase the utilization and thus decrease the amount of available slack. This can eventually exhaust all the available slack in the system. If this condition occurs, the execution of AP(k+1) will not be possible, therefore, the INCA Server will stop its execution.

During the execution of the some $\operatorname{AP}(k)$ algorithm, a new task may arrive in the system or a task may leave the system. If this occurs, the INCA Server will re-start its execution, taking into account the modified load of the system. If the incremental server is invoked when a task leaves the system, the instructions in lines 3, 4, 5, 6 (from Figure 1) are not executed.

4. Stopping the execution of the server. The conditions for finishing the execution of the server are a). there is no more slack in the schedule to execute some $\operatorname{AP}(k)$ algorithm, or b). the result of $A P(k)$ is not better than the result of $A P(k-1)$. Also, after $A P(n)$ is executed the server finish its execution.

\subsection{The Approximate Algorithm: $A P(k)$}

In this section, we describe the approximate algorithm used by the INCA Server. The approximate algorithm makes use of a greedy-type procedure[16] which finds a heuristic solution by selecting for execution optional parts in order of decreasing utilization $\frac{p_{i}}{T_{i}}$ if the objective function is $\mu(s)$, or $\frac{v_{i}}{p_{i} / T_{i}}$ if the objective function is $\gamma(s)$ [16]. The algorithm $\mathrm{AP}(\mathrm{k})$ considers all possible subsets in the search space with at least $k$ optional parts chosen for execution. It first chooses for inclusion in the schedule a subset of $k$ optional parts, and if this subset does not satisfy our feasibility condition (UBT) it is discarded and a new subset with $k$ optional parts is selected. If the subset passes the UBT, the remaining optional parts are considered for selection in decreasing order of $\frac{p_{i}}{T_{i}}$ or $\frac{v_{i}}{p_{i} / T_{i}}$, while the UBT is satisfied. The best solution obtained by examining all subsets of $k$ optional parts is the solution generated by this algorithm.

have decided to schedule the new task at the end of the last period of the instances running in the system when the new task arrives. 


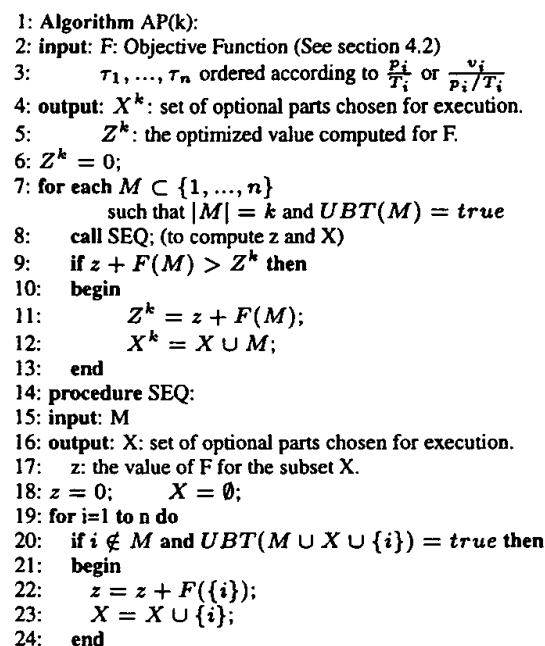

Figure 2. Approximate Algorithm (AP)

The algorithm is described in Figure 2. The output of algorithm $\mathrm{AP}(\mathrm{k})$ is $X^{k}$ and $Z^{k}$. $X^{k}$ denotes the set of optional parts chosen for execution and $Z^{k}$ denotes the optimal solution found by $\mathrm{AP}(\mathrm{k})$. Note that $Z^{k}$ approximates $U^{\max }$ or $V^{\max }$ depending on the objective function used.

The time complexity of procedure SEQ is $O(n)$ : there is a loop for each task, and the UBT can be computed incrementally in $O(1)$ for each task. Since the number of times SEQ is executed is $O\left(n^{k}\right)$, the time complexity of $\mathrm{AP}(\mathrm{k})$ is $O\left(n^{k+1}\right)$. Even for a small number of tasks (e.g., $n=10$ tasks) this complexity seems rather high. However, we will demonstrate with simulations that for $k \leq 2$, the value of the system is very close to optimal. The worst-case performance ratio of $\mathrm{AP}(\mathrm{k})$ is $\frac{k}{k+1}[16]$, which directs us to a solution with large $k$. However, since the complexity of the $\operatorname{AP}(k)$ algorithm is high for large $k$, we are interested in finding the smallest value of $k$ such that $\mathrm{AP}(\mathrm{k})$ reaches a near-optimal solution. In the following example we will measure the real performance of the AP(k) algorithm in terms of complexity and run-time.

Example 1: Consider the set of tasks with its associated timing constraints and criticality values described in Table 2 . Our goal is to apply the approximate algorithm for our objective functions and to evaluate its performance. The total utilization of the set of tasks in Table 2 denotes an overload (load =120\%). The utilization of the mandatory parts is $54 \%$ and the utilization of the optional parts is $66 \%$. The problem to be solved is to handle the overload for this workload using the AP(k) algorithms, selecting the number of task to be included for execution such that our optimality criteria is satisfied. Tables 3 and 4 show the results from algorithm $\mathrm{AP}(\mathrm{k})$, for $k=0, \ldots, 5$. The results shown in the Tables are: (a) the result from al- gorithm $\mathrm{AP}(\mathrm{k})$, which is $X^{k}$ and $Z^{k}$ (see Figure 2); (b) the number of combinations (N.C) necessary to obtain a solution; (c) the run-time of $\mathrm{AP}(\mathrm{k})$, which denotes the physical time in microseconds, using a PC Intel $233 \mathrm{MHz}$ running Linux with $48 \mathrm{MB}$ of RAM; and (d) the set of optional parts chosen for execution $\left(X^{k}\right)$

\begin{tabular}{|c|cccccc|}
\hline Task & $C_{i}$ & $T_{i}$ & $m_{i}$ & $p_{i}$ & $\boldsymbol{u}_{i}$ & Value \\
\hline$\tau_{1}$ & 39.0 & 116.0 & 18.0 & 21.0 & 0.336 & 37.0 \\
\hline$\tau_{2}$ & 49.0 & 154.0 & 23.0 & 26.0 & 0.318 & 30.0 \\
\hline$\tau_{3}$ & 44.0 & 174.0 & 18.0 & 26.0 & 0.253 & 27.0 \\
\hline$\tau_{4}$ & 47.0 & 195.0 & 20.0 & 27.0 & 0.241 & 29.0 \\
\hline$\tau_{5}$ & 47.0 & 903.0 & 27.0 & 20.0 & 0.052 & 2.0 \\
\hline
\end{tabular}

Table 2. Example Real-Time Workload: Mandatory and Optional parts and Criticality Values

\begin{tabular}{|c|cccc|}
\hline $\mathrm{k}$ & $Z^{k} \approx U^{\max }$ & $($ N.C) & Run-Time $(\mu \mathrm{s})$ & Result Set \\
\hline 0 & 89.030136 & 4 & 16 & 11000 \\
\hline 1 & 91.244980 & 16 & 33 & 11001 \\
\hline 2 & 91.244980 & 24 & 44 & 11001 \\
\hline 3 & 99.715370 & 17 & 38 & 01110 \\
\hline 4 & 99.715370 & 5 & 14 & 01110 \\
\hline 5 & 99.715370 & 1 & 6 & 01110 \\
\hline
\end{tabular}

Table 3. Results for maximizing utilization.

\begin{tabular}{|c|cccc|}
\hline $\mathrm{k}$ & $Z^{k} \approx V^{\max }$ & $($ N.C) & Run-Time $(\mu \mathrm{s})$ & Result Set \\
\hline 0 & 0.49 & 4 & 13 & 10010 \\
\hline 1 & 0.492 & 16 & 36 & 10011 \\
\hline 2 & 0.559 & 25 & 58 & 01110 \\
\hline 3 & 0.559 & 17 & 46 & 01110 \\
\hline 4 & 0.559 & 5 & 18 & 01110 \\
\hline 5 & 0.559 & 1 & 7 & 01110 \\
\hline
\end{tabular}

Table 4. Results for maximizing criticality.

For the goal of maximizing utilization, it is possible to observe that $\mathrm{AP}(\mathrm{k})$ with $k=3$ yields optimal results, while for maximizing criticality $\mathrm{AP}(\mathrm{k})$ for $k=2$ yields the optimal solution. For the case of utilization, tasks are ordered in terms of decreasing $\frac{p_{i}}{T_{i}}$ (i.e., $\tau_{1}, \tau_{2}, \tau_{3}, \tau_{4}, \tau_{5}$ ), while for the case of the criticality the order is by decreasing $\frac{v_{i}}{p_{i} / T_{i}}$ (i.e., $\tau_{4}, \tau_{1}, \tau_{3}, \tau_{2}$, $\left.\tau_{5}\right)$.

\subsubsection{Measuring the Performance and Complexity of the Solutions}

To extend the previous results, an experiment with 1000 randomly generated task sets has been conducted for measuring the performance and the complexity of the algorithm. For each experiment, a workload of 10 tasks has been generated with an overload ( $120 \%$ utilization for each task set). Results shown in Table 5 indicate the number of solutions within a certain percent close to optimal. For the two optimality criteria a near optimal solution (more than $91 \%$ ) is obtained using $A P(2)$. For example, for maximizing utilization, results for $A P(2)$ indicate that 951 experiments yield a near optimal solution (0$0.1 \%$ ) and the remaining 49 yield a $1-5 \%$ near optimal solution. 

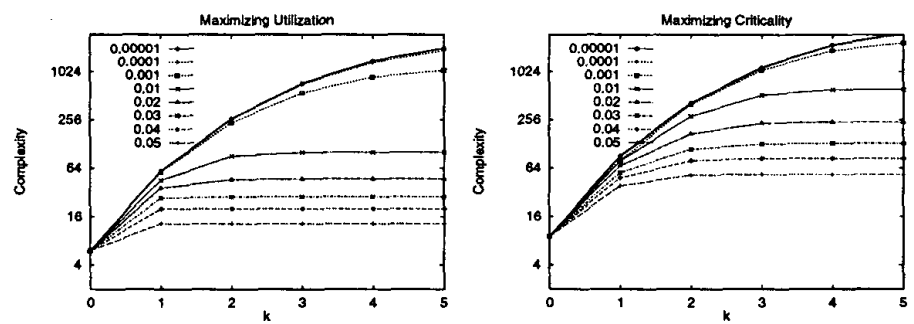

Figure 3. Complexity of $\operatorname{AP}(k)$ for different values of $\epsilon . Y$ axis is in log scale.
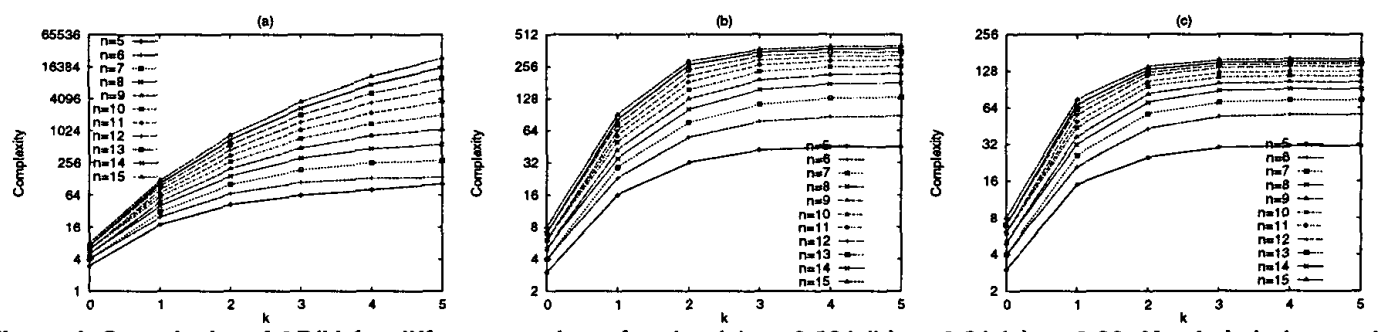

Figure 4. Complexity of $\operatorname{AP}(k)$ for different number of tasks, (a) $\epsilon=0.001$ (b) $\epsilon=0.01$ (c) $\epsilon=0.02$. $Y$ axis is in log scale.

For maximizing criticality, results show that for AP(2) 911 experiments yield a near optimal solution $(0-0.1 \%)$. This surprising result shows the excellent performance of the approximate algorithm $\mathrm{AP}(\mathrm{k})$.

\begin{tabular}{|c|ccccc|}
\hline & \multicolumn{5}{|c|}{ Maximizing Utilization } \\
\hline$k$ & $0-0.1 \%$ & $1-5 \%$ & $5-10 \%$ & $10-15 \%$ & $15-20 \%$ \\
\hline 0 & 617 & 251 & 119 & 13 & 0 \\
\hline 1 & 662 & 336 & 2 & 0 & 0 \\
\hline 2 & 951 & 49 & 0 & 0 & 0 \\
\hline 3 & 999 & 1 & 0 & 0 & 0 \\
\hline 4 & 1000 & 0 & 0 & 0 & 0 \\
\hline \hline & \multicolumn{7}{|c|}{} & \multicolumn{4}{|c|}{ Maximizing Criticality } \\
\hline 0 & 631 & 131 & 62 & 50 & 83 \\
\hline 1 & 829 & 35 & 54 & 51 & 28 \\
\hline 2 & 911 & 15 & 25 & 25 & 24 \\
\hline 3 & 1000 & 0 & 0 & 0 & 0 \\
\hline 4 & 1000 & 0 & 0 & 0 & 0 \\
\hline
\end{tabular}

Table 5. Number of Solutions within $x$ percent near optimal for 1000 Tasks Sets.

Further reductions in complexity could be obtained by relaxing the feasibility bound (see UBT). According to our feasibility tests, an element $s \in S$ is feasible if its feasibility condition is met. However a result less than $100 \%$ (e.g., 95\%) could be sufficient for some applications which would cause an earlier end to the search for feasible solutions. Let us define $\epsilon, 0<\epsilon<1.0$, as the feasibility error which indicates a relaxation on the feasibility condition. The feasibility test shown in Equation (5) indicates a sufficient feasibility condition.

$$
\sum_{i=1}^{n} \frac{m_{i}}{T_{i}}+\frac{x_{i} p_{i}}{T_{i}} \leq 1.0-\epsilon
$$

We are interested in measuring the complexity (the number of elements searched) of the algorithm using the sufficient feasibility condition for different values of $\epsilon$. We have conducted 1000 experiments comprising 10 tasks in each experiment whose total utilization (mandatory + optional) is 1.2. The average complexity of the algorithm is shown in Figure 3 for a varying value of $\epsilon$. Note that the complexity of our algorithm is much lower than the worst-case complexity (exemplified by $\epsilon=10^{-5}$ ) and that big reductions in complexity can be achieved by increasing the value of $\epsilon$. For example for $\epsilon=0.02$ and $k=5$ the complexity achieved is 47 and 240 for maximizing utilization and criticality, respectively. However, it is worth noting that for all values of $k$ the algorithm for maximizing utilization performs slightly better than for maximizing criticality. A possible explanation for this surprising result is the fact that the number of tasks is relatively low $(n=10)$. According to the worst-case complexity of the algorithm, having a higher number of tasks may increase considerably the complexity of the algorithm.

To measure the effect of the number of tasks on the complexity of the algorithm, an experiment has been conducted using 1000 randomly generated tasks sets for three values of $\epsilon=0.001, \epsilon=0.01, \epsilon=0.02$ and for a varying number of tasks (from 5 to 15 tasks). Figure 4 shows that the complexity of the algorithm is relatively low even with a high degree of quality $(\epsilon=0.02)$.

From the results shown in Table 5 it can be concluded that for values of $k \leq 2,92.5 \%$ and $100 \%$ of the solutions are $95 \%$-close to optimal when the criterion is to maximize criticality and utilization, respectively. Note that when $k=1$, only $0.2 \%$ are less than $95 \%$ of optimal for the utilization criterion. From Figures 3 and 4, it can be seen that keeping $\epsilon$ between the values of 0.0001 and 0.02 is reasonable for achieving low complexity while maintaining high quality results. 


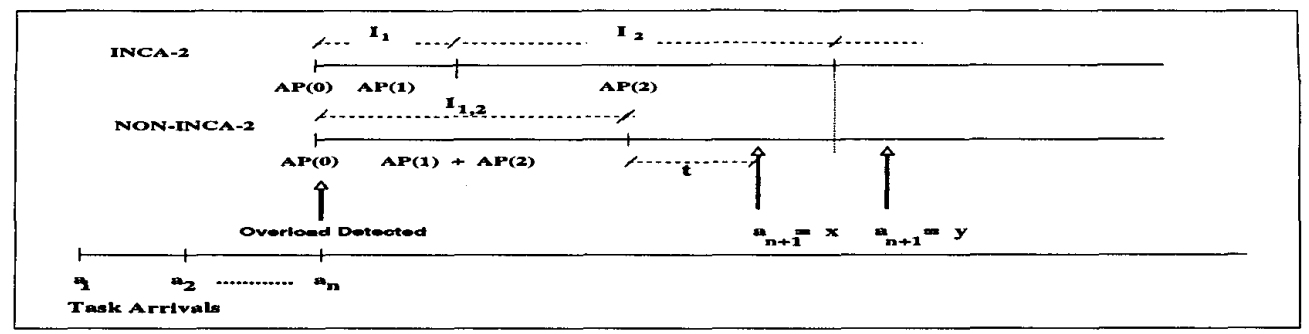

Figure 5. INCA-2 and NON-INCA-2 Servers: Execution Sequences.

\section{Analysis of the INCA Server}

As explained above, the INCA server is based on the incremental execution of several stages (Approximate Algorithms). At the end of each stage, information is available regarding the optional parts chosen for execution, and the resulting value of the objective function for the set of optional parts chosen. The process of scheduling the chosen optional parts at every stage will be called commitment. The INCA server executes the approximate algorithm $\mathrm{AP}(\mathrm{k})$ only after $A P(k-1)$ commits. In contrast, a non-incremental server would execute a number of stages $A P(0), \ldots, A P(k)$, before committing to the system.

In what follows we analyze the merit of the incremental execution by assuming $k=2$ and comparing the following two servers,

INCA-2: This is the incremental execution sequence used by the INCA Server considering only two stages. That is, $A P(1)$, commit, $A P(2)$, commit.

NON-INCA-2: In this case, there is no incremental execution, and two stages are executed continuously before committing. That is, $A P(1)+A P(2)$, commit.

In Figure 5 we illustrate a sequence of $n$ tasks arriving in the system, where the arrival of task $\tau_{n}$ causes an overload. $a_{n+1}=x$ and $a_{n+1}=y$ denote two instants of time at which a new tasks $\tau_{n+1}$ may arrive. Although the following analysis considers only two stages of the execution of the incremental server, the same analysis can be easily extended to include more than 2 stages.

After executing $\mathrm{AP}(0)$ at time $a_{n}, X^{0}$ and $Z^{0}$ are obtained. The set of optional parts, $X^{\mathbf{0}}$, selected for execution will execute until AP(1) commits. Then a new set of optional parts $X^{1}$ will be chosen for execution and the process repeats. The resulting utilization or criticality value, $Z^{0}$, will depend on the objective function used. Let the utilization after committing at $\mathrm{AP}(\mathrm{k})$ be: $Z^{k}=\left(1-\alpha_{k}\right)$, where $\alpha_{k}$ denotes the resulting slack time expressed in a percentage of resource usage. This slack time will be used for the execution of $\mathrm{AP}(\mathrm{k}+1)$. Note that $\alpha_{k+1} \leq \alpha_{k}$ if the objective of the $\mathrm{AP}(\mathrm{k})$ algorithm is to maximize utilization.

Definition 1. Let $\phi_{k}$ be the worst-case execution time of algorithm $\mathrm{AP}(\mathrm{k})$ measured continuously (i.e., without interference from other tasks). We assume that the execution time of $\operatorname{AP}(0)$, $\phi_{0}$, is negligible.

Definition 2. The interval of time at which $\mathrm{AP}(\mathrm{k})$ executes is defined as $I_{k}=\frac{\phi_{k}}{\alpha_{k-1}}$. If the execution of $\mathrm{AP}(1)$ and AP(2) is non incremental, the interval of time at which both $\mathrm{AP}(1)+\mathrm{AP}(2)$ execute is defined as $I_{1,2}=\frac{\phi_{1}+\phi_{2}}{\alpha_{0}}$ (see Figure $5)$.

Utilization Metric We will compare the cumulative utilization achieved by INCA- 2 and NON-INCA- 2 during the period from $a_{n}$ to $a_{n+1}$. If during a period $I$, the slack in the system is a constant, $\alpha$, then the cumulative utilization given by Equation 1 can be alternatively computed from $C U(I)=I(1-\alpha)$. Given that both INCA-2 and NON-INCA-2 will produce a utilization of $\left(1-\alpha_{0}\right)$, resulting from $\mathrm{AP}(0)$, during the period $\left[a_{n}, a_{n}+I_{1}\right]$, and that both will produce a utilization of $\left(1-\alpha_{2}\right)$; resulting from $\mathrm{AP}(2)$ during the period $\left[a_{n}+I_{1}+I_{2}, a_{n+1}\right]$, if $a_{n+1}>\left(a_{n}+I_{1}+I_{2}\right)$, we will only compare the utilizations during the period $\left[a_{n}+I_{1}, a_{n+1}\right]$, where $a_{n+1} \leq a_{n}+I_{1}+I_{2}$.

We will denote the cumulative utilization resulting from INCA- 2 by $C U$ while denoting the cumulative utilization resulting from NON-INCA-2 by $C U_{N}$. The following lemma proves that the incremental server always outperforms the non incremental server when the goal is to increase the cumulative utilization of the system.

Lemma 1. $C U\left(\left[a_{n}, a_{n+1}\right]\right) \geq C U_{N}\left(\left[a_{n}, a_{n+1}\right]\right)$, if the objective of the $\mathrm{AP}(\mathrm{k})$ algorithm is to maximize utilization.

Proof: If $a_{n+1} \leq a_{n}+I_{1}$ then both servers produce the same utilization, while if $a_{n}+I_{1} \leq a_{n+1} \leq a_{n}+I_{1,2}$ then the INCA server is at least as good as the NON-INCA server, since $C U\left(\left[a_{n}+I_{1}, a_{n}+I_{1,2}\right]\right)=\left(I_{1,2}-I_{1}\right)\left(1-\alpha_{1}\right)$ and $C U_{N}\left(\left[a_{n}+I_{1}, a_{n}+I_{1,2}\right]\right)=\left(I_{1,2}-I_{1}\right)\left(1-\alpha_{0}\right)$.

Now assume that $a_{n+1}=a_{n}+I_{1,2}+t$, where $0 \leq t \leq$ $I_{1}+I_{2}-I_{1,2}$. In this case,

$$
\begin{aligned}
C U\left(\left[a_{n}, a_{n+1}\right]\right) & =I_{1}\left(1-\alpha_{0}\right)+I_{2}\left(1-\alpha_{1}\right) \\
C U_{N}\left(\left[a_{n}, a_{n+1}\right]\right) & =I_{1,2}\left(1-\alpha_{0}\right)+t\left(1-\alpha_{2}\right)
\end{aligned}
$$

using the values of $I_{1}, I_{2}$ and $I_{1,2}$ from Definition 2 we get $C U\left(\left[a_{n}, a_{n+1}\right]\right)=$

$$
C U_{N}\left(\left[a_{n}, a_{n+1}\right]\right)+\frac{\phi_{2}}{\alpha_{1}}-\frac{\phi_{2}}{\alpha_{0}}-t\left(1-\alpha_{2}\right)
$$


Given that $t \leq I_{1}+I_{2}-I_{1,2}=\frac{\phi_{2}}{\alpha_{1}}-\frac{\phi_{2}}{\alpha_{0}}$, and that $\left(1-\alpha_{2}\right) \leq$ 1 , we conclude that $C U\left(\left[a_{n}, a_{n+1}\right]\right)$ is equal to or larger than $C U_{N}\left(\left[a_{n}, a_{n+1}\right]\right)$. Finally, if $a_{n+1}>a_{n}+I_{1}+I_{2}$, then INCA2 and NON-INCA-2 produce the same utilization, $\left(1-\alpha_{2}\right)$, for any period latter than $a_{n}+I_{1}+I_{2}$ and thus the result that $C U\left(\left[a_{n}, a_{n+1}\right]\right) \geq C U_{N}\left(\left[a_{n}, a_{n+1}\right]\right)$ holds.

QED

Criticality Metric The above lemma assumes that the objective of the servers is to maximize the system utilization. If however, the goal is to maximize the cumulative criticality (see Equation 2), then the relative performance of INCA-2 and NON-INCA-2 depends on the performance of the incremental algorithms $\mathrm{AP}(0), \operatorname{AP}(1)$ and $\mathrm{AP}(2)$. Let $Z^{k}$ be the criticality value achieved by $A P(k)$, when the goal of $A P(k)$ is to maximize criticality, and let $\alpha_{k}$ be the slack of the system after $\mathrm{AP}(\mathrm{k})$ commits. As before, we will use $C V(I)$ and $C V_{N}(I)$ to denote the cumulative criticality obtained by INCA-2 and NON-INCA-2 respectively during the period $I$.

Lemma 2. If $a_{n+1} \leq I_{1,2}$, then $C V\left(\left[a_{n}, a_{n+1}\right]\right) \geq$ $C V_{N}\left(\left[a_{n}, a_{n+1}\right]\right)$, when the objective of $A P(k)$ is to maximize criticality.

Proof: $C V\left(\left[a_{n}, a_{n}+I_{1}\right]\right)=C V_{N}\left(\left[a_{n}, a_{n}+I_{1}\right]\right)=I_{1} * Z^{0}$, while for $a_{n}+I_{1} \leq t \leq a_{n+1}$, we have

$$
\begin{aligned}
C V\left(\left[a_{n}+I_{1}, a_{n}+I_{1}+t\right]\right) & =t * Z^{1} \\
C V_{N}\left(\left[a_{n}+I_{1}, a_{n}+I_{1}+t\right]\right) & =t * Z^{0}
\end{aligned}
$$

The result follows since $Z^{1} \geq Z^{0}$.

QED

Lemma 3. If $a_{n+1}>I_{1}+I_{2}$ and the objective of $A P(k)$ is to maximize criticality, then $C V\left(\left[a_{n}, a_{n+1}\right]\right) \geq$ $C V_{N}\left(\left[a_{n}, a_{n+1}\right]\right)$ if and only if $\alpha_{1}\left(Z^{2}-Z^{1}\right) \leq \alpha_{2}\left(Z^{2}-Z^{0}\right)$.

Proof: Both INCA-2 and NON-INCA-2 produce the same criticality, namely $Z^{2}$ after the time $I_{1}+I_{2} . Z^{2}=C V\left(\left[I_{1}+\right.\right.$ $\left.\left.I_{2}, a_{n+1}\right]\right)=C V_{N}\left(\left[I_{1}+I_{2}, a_{n+1}\right]\right)$. Hence, we will only compare the cumulative criticality in the period from $a_{n}+I_{1}$ to $a_{n}+I_{1}+I_{2}$.

$C V\left(\left[a_{n}+I_{1}, a_{n}+I_{1}+I_{2}\right]\right)=I_{2} * Z^{1}$

$C V_{N}\left(\left[a_{n}+I_{1}, a_{n}+I_{1}+I_{2}\right]\right)=$

$$
\left(I_{1,2}-I_{1}\right) * Z^{0}+\left(I_{1}+I_{2}-I_{1,2}\right) * Z^{2}
$$

Substituting for the values of $I_{1}, I_{2}$ and $I_{1,2}$ we get $C V\left(\left[a_{n}+I_{1}, a_{n}+I_{1}+I_{2}\right]\right)=\frac{\phi_{2}}{\alpha_{1}} * Z^{1}=$

$C V_{N}\left(\left[a_{n}+I_{1}, a_{n}+I_{1}+I_{2}\right]\right)=\frac{\phi_{2}}{\alpha_{0}} * Z^{0}+\left(\frac{\phi_{2}}{\alpha_{1}}-\frac{\phi_{2}}{\alpha_{0}}\right) * Z^{2}$

Hence, $C V\left(\left[a_{n}+I_{1}, a_{n}+I_{1}+I_{2}\right]\right)=C V_{N}\left(\left[a_{n}+I_{1}, a_{n}+\right.\right.$ $\left.\left.I_{1}+I_{2}\right]\right)+\frac{\phi_{2}}{\alpha_{0}}\left(Z^{2}-Z^{0}\right)-\frac{\phi_{2}}{\alpha_{1}}\left(Z^{2}-Z^{1}\right)$.

The lemma follows directly from the last Equation. QED

Given that $\mathrm{AP}(\mathrm{k})$ does not decrease the value of $Z^{k}$ bellow $Z^{k-1}$, then $\left(Z^{2}-Z^{1}\right) \leq\left(Z^{2}-Z^{0}\right)$. However, nothing can be said about the relative values of $\alpha_{1}$ and $\alpha_{2}$ if $\operatorname{AP}(\mathrm{k})$ is used to improve criticality rather than utilization. The relative performance of the INCA and NON-INCA servers will be studied using simulations in the next section.

\section{Simulation Experiments}

The following simulation experiments have been designed to test the performance of the incremental server and its ability to achieve our optimality criteria using synthetic workloads. We are interested in measuring the performance of the algorithm using up to five stages of execution. According to the results obtained in Section 5.2 we are aware that statically we need to execute no more that 3 stages to achieve near-optimal results. Our goals are the following:

- to measure the quality of the results over a large set of dynamic tasks that arrive and leave the system at arbitrary instants of time.

- to measure and compare the performance among several stages for our different optimality criteria.

Each plot on the graphs represents the average of a set of 100 independent simulations. Up to the first 5 stages of the INCA server are executed in each simulation. Each curve INCA- $k$ in the graphs denotes the execution of the INCA server in which only the first $k$ stages are executed. That is, only the incremental execution of $A P(j)$, for $j=0, \ldots, k$ is considered.

On each simulation 5,000 tasks are generated dynamically. Each task has a life-time $\left(l_{i}\right)$ that follows a uniform distribution between 400 and 600 instances (periods). At the end of its life-time, the task leaves the system. The utilization of task $\tau_{i}, U_{i}$, is chosen as a random variable with uniform distribution between $5 \%$ and $20 \%$. The period $T_{i}$ of each task is chosen as a random variable with uniform distribution between 30 and 100 time units. The computation time of task $\tau_{i}$ is $C_{i}=T_{i} * U_{i}$. The experiments were conducted with a total utilization $U_{T}=\sum_{i} \frac{C_{i}}{T_{i}}$ varying between $80 \%$ and $180 \%$. The number of tasks in the system $(n t)$ executing at any time is computed by $n t=\frac{U_{r}}{U_{i}}$. The task inter-arrival time is computed by $I T_{i}=\frac{T_{i} * l t_{i}}{n t}$. The computation time of the optional part $p_{i}$ is a random variable that follows a uniform distribution between $40 \%$ and $60 \%$ of the total computation time of task $\tau_{i}$.

The execution time of $\mathrm{AP}(\mathrm{k})$ used was obtained from the experiments described by Figure 5, using a value of $\epsilon=0.001$. Throughout this simulation experiments we will consider randomly generated correlated tasks sets [15], which means that the criticality is a linear function of the utilization ${ }^{7}$. The value $v_{i}$ of each task is randomly distributed in $\left[U_{i}-0.10, U_{i}+0.10\right]$ such that $v_{i}>0$ (i.e., plus or minus $10 \%$ from the utilization of the task).

The performance of our algorithms was measured according to the following metrics:

\footnotetext{
${ }^{7}$ It is hard to maximize the criticality value ratio for correlated tasks sets because many task combinations give similar results, therefore a larger number of combinations must be computed in order to find an optimal solution. We do not consider uncorrelated task sets because it is relatively easier to maximize their criticality value ratio (there is a large variation between the utilization of the tasks, making it easier to obtain a feasible and optimal solution).
} 

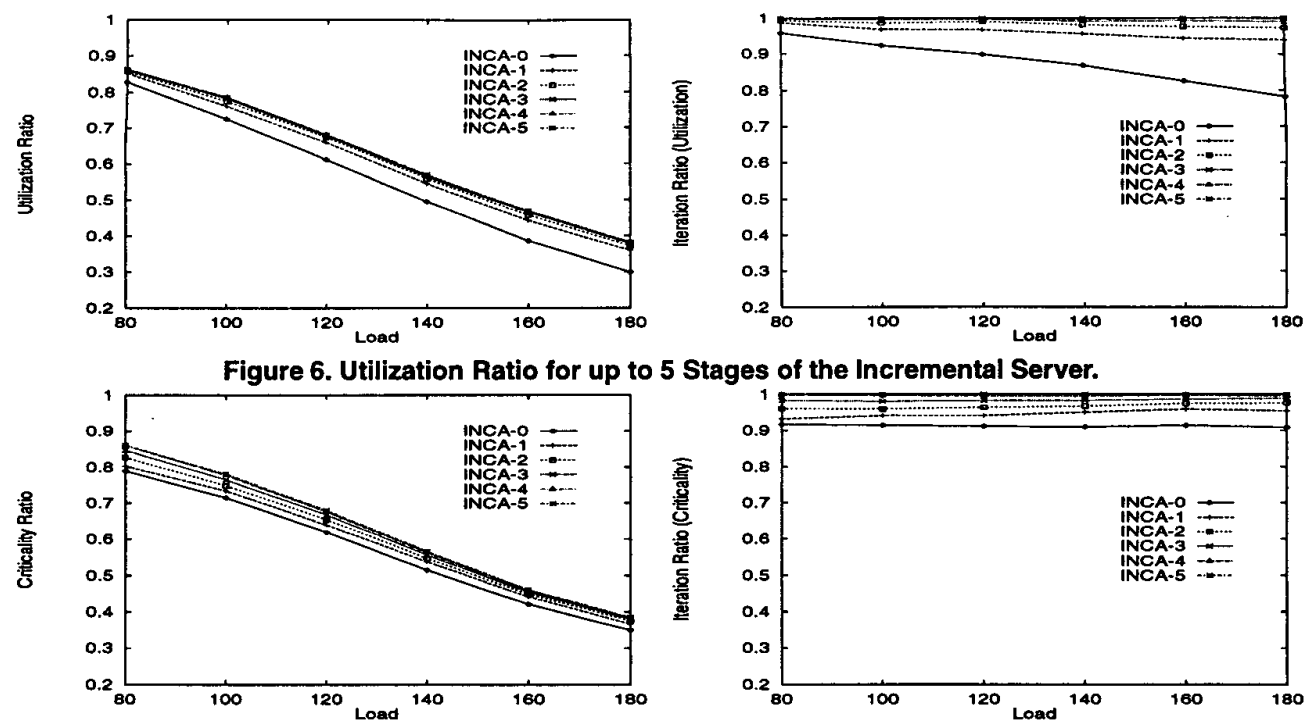

Figure 7. Criticality Ratio for up to 5 Stages of the Incremental Server.
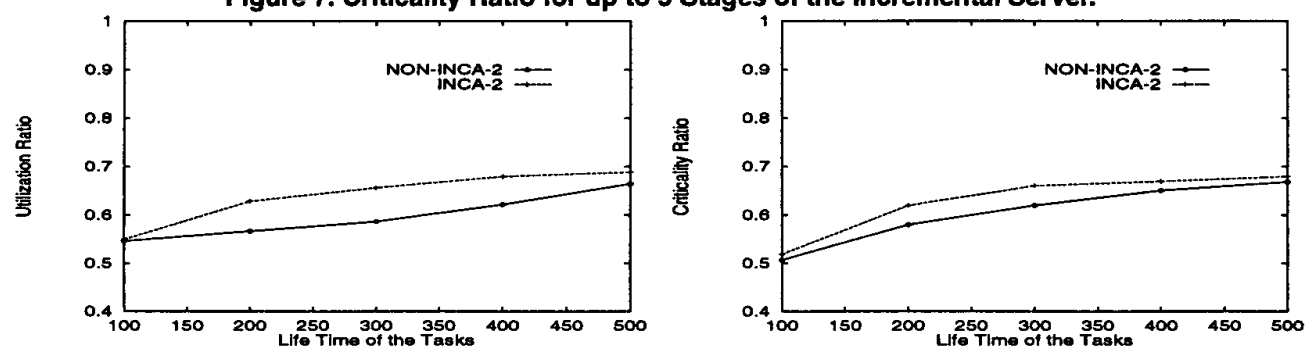

Figure 8. Utilization and Criticality Ratio for the INCA and NON-INCA Servers.

- Utilization Ratio: This metric is computed as follows,

$$
\text { Utilization Ratio }=\frac{C U(I)}{\text { Total Utilization }}
$$

where $C U(I)$ is the cumulative utilization of the system (see Section 4), for the interval of time $I$ that denote the total duration of the schedule. The total utilization that can be achieved is computed by: $\sum_{i} r_{i} * p_{i}$, where the sum is over all tasks that arrive to the system in the interval of time $I$. Recall that $r_{i}$ denotes the total number of instances of $\tau_{i}$.

- Criticality Ratio: This metric is computed as follows,

$$
\text { Criticality Ratio }=\frac{C V(I)}{\text { Total Criticality }}
$$

where $C V(I)$ is the cumulative criticality (see Section 4), for the interval of time $I$ that denote the total duration of the schedule. The total criticality that can be achieved is computed by, $\sum_{i} r_{i} * v_{i}$.

Two sets of experiments were conducted for our simulations. The first experiment, shown in Figures 6 and 7 was designed to compare the performance of INCA-k for different values of $k, 0 \leq k \leq 5$. The second experiment, shown in Figure 8 was designed to compare the performance of the INCA-2 algorithm against the NON-INCA-2 algorithm. In the graphs shown in Figures 6 and 7, the utilization and the criticality ratio were measured. The left graph shows the value of the utilization metric, while the graph on the right shows the ratio of the value obtained by INCA-k and INCA-5, called iteration ratio.

The results shown in Figures 6 and 7 indicate that for values of $k>2$ there is no significant improvement on the performance of the INCA server. Therefore, we will consider that INCA-5 achieves the maximum value possible in the system. For the utilization ratio, it is observed that INCA-2 achieves results close to those obtained by INCA- 5 for all load values. Notice that for the iteration ratio, Figure 6 shows that INCA-0 achieves a performance that varies from $96 \%$ of INCA- 5 for a load of 0.80 , to $78 \%$ of INCA- 5 for a load of 1.80 . The performance of the algorithm for INCA-2 varies from $99 \%$ to $98.5 \%$ of INCA-5. It is important to note that even INCA-1 achieves a utilization performance higher or equal to $95 \%$ of INCA-5. The performance results for the criticality ratio indicate that INCA-k $(k=0, \ldots, 4)$ yield a performance higher than $90 \%$ of INCA-5 for all values of the load. 
For our second experiment, Figure 8 shows the utilization and criticality ratio for the INCA-2 and the NON-INCA-2 servers. Our main interest in this experiment is to validate the results obtained previously in the analysis of the INCA server (see Section 6). In this experiment, the load of the system has a fixed value of $120 \%$, and the life-time of each task $l t_{i}$ varies between 100 and 500 .

The behavior of the INCA-2 and NON-INCA-2 servers can be explained as follows: For low values of $l t_{i}$ (e.g., 100 instances) both servers yield similar values because both servers are only able to execute $\mathrm{AP}(0)$ (which removes the overload). For $l t_{i}=200, \ldots, 300$ the INCA-2 server yields much better results, because the INCA-2 server is capable of committing more frequently than NON-INCA-2. In this situation, the NON-INCA-2 server is able to execute a few times AP(1) and $\operatorname{AP}(2)$ but is mostly only able to execute $\mathrm{AP}(0)$. Finally, for the last values of $l t_{i}$ (e.g. 400 and 500 instances) the performance of INCA-2 and NON-INCA-2 servers get closer because both servers are now able to commit both $\mathrm{AP}(1)$ and $\mathrm{AP}(2)$. In any case, the performance of the INCA- 2 server is better that that of the NON-INCA server for all values of $l t_{i}$.

The results obtained in our simulations confirm the results obtained Section 6 and indicate that the INCA Server is a low cost and effective mechanism for scheduling real-time tasks under overloaded conditions.

\section{Conclusion}

In this paper, the problem of scheduling an overloaded realtime system has been studied. As observed by different research studies $[8,4,9,11]$, a significant performance degradation may occur in the system if the overload is not addressed efficiently. The set of tasks selected for execution is crucial for the proper operation of an overloaded real-time system. In our framework, each task has an assigned criticality value, and an objective function is evaluated in overloaded conditions such that an optimality criteria is met. The process of selecting tasks to discard while meeting the optimality criteria requires the exploration of a potentially large number of combinations. Since this process is too time consuming to be computed on-line, we have developed an Incremental Server (INCA) scheduling paradigm, which is based in a sequence of approximate algorithms. The execution of the approximate algorithms is conducted in an incremental manner, during the time at which the processor would otherwise be idle (slack-time), progressively refining the quality of the solution. The computational complexity of the INCA Server is high. However, we have shown that in practice only few stages need to be executed for achieving near-optimal solutions. An important feature of the incremental algorithm is that its run-time overhead and the quality of the solutions are parameters that can be controlled on-line. Our simulation results show that our approximate algorithm is efficient, has low overhead, and most importantly generates near-optimal solutions for overloaded real-time systems.

\section{References}

[1] H. Aydın, R. Melhem, D. Mossé, P. Mejía-Alvarez. “Optimal Reward-Based Scheduling of Periodic Real-Time Tasks", Proc. of the IEEE Real Time Systems Symposium, Dec. 1999.

[2] A. Burns, D. Prasad, A. Bondavalli, F.Di. Giandomenico, K. Ramamritham, J. Stankovic, L. Strigini "The Meaning and Role of Value in Scheduling Flexible Real-Time Systems", J. of Systems Architecture, Jan. 2000

[3] A. Burns and D. Prasad, "Value-Based Scheduling of Flexible Real-Time Systems for Intelligent Autonomous Vehicle Control", Proc. of the 3rd. IFAC Symposium on Intelligent Autonomous Vehicles March 1998.

[4] G.C. Butazzo, "Red: A Robust Earliest Deadline Scheduling Algorithm", Proc. of Third Int. Workshop on Responsive Computing Systems, Spain, Dec. 1998.

[5] G.C. Butazzo, "Hard Real-Time Computing Systems: Predictable Scheduling Algorithms and Applications", Kluwer Academic Publisher, 1997.

[6] H. Chetto and M. Chetto. "Some Results of the Earliest Deadline Scheduling Algorithm", IEEE Transactions on Software Engineering, Oct. 1989.

[7] M. Hamdaoui, P. Ramanathan. "A Dynamic Priority Assignment Technique for Streams with (m,k)-firm Deadlines", IEEE Transactions on Computers, Dec. 1995.

[8] S. Hwang, C.M. Chen and A.K. Agrawala. "Scheduling an Overloaded Real-Time System", Proc. of the 1996 IEEE Conference on Computers and Communications.

[9] E.D. Jensen, J.D. Northcutt, R.K.Clark, S.E. Shipman, F.D. Reynolds, D.P. Maynard, K.P. Loepfere. "Alpha: An Operating System for the Mission-Critical Integration and Operation of Large, Complex, Distributed Real-Time Systems - An Overview", OSMCC, Sept. 1989.

[10] G. Koren and D. Shasha. "Skip-over: Algorithms and Complexity for Overloaded Real-Time Systems", Proc. of the IEEE Real Time Systems Symposium, Dec. 1995.

[11] G. Koren and D. Shasha. "D-over: An Optimal Scheduling Algorithm for Overloaded Real-Time Systems", Proc. of the IEEE Real Time Systems Symposium, 1992.

[12] C.L. Liu, J. Layland. "Scheduling Algorithms for Multiprogramming in Hard Real-Time Environments", J. ACM 20(1). pp. 46-61, Jan. 1973.

[13] J.W. Liu and W.K. Shih. "Imprecise Computations", Proceedings of the IEEE, Jan. 1994.

[14] C.D. Locke. "Best-effort Decision Making for Real-Time Scheduling", PhD. Thesis, CS-CMU. 1986

[15] S. Martello, P. Toth. "Knapsack Problems", Wiley, 1990

[16] S. Sahni. "Approximate Algorithms for the 0/1 Knapsack Problem", J. of the ACM, Jan. 1975. 